\title{
ON PROJECTIVE REPRESENTATIONS OF CERTAIN FINITE GROUPS ${ }^{1}$
}

\author{
CHARLES W. CURTIS
}

Introduction. In this paper it is proved that the irreducible projective representations of the group $G$ of automorphisms of a Lie algebra of classical type constructed in [2] remain irreducible and inequivalent when restricted to the subgroup $G_{0}$ of $G$ generated by the oneparameter subgroups $\left\{\exp \left(a d \xi e_{\alpha}\right)\right\}$ where $\alpha$ ranges over the set of roots of $\mathfrak{R}$ with respect to a fixed Cartan subalgebra, and $\xi$ is taken from the prime field $\Omega_{0}$ in $\Omega$. The proofs of irreducibility and inequivalence given in [2] apply without change to $G_{0}$ in case $\Omega_{0}$ is infinite, so there is no problem unless $\Omega_{0}$ is the prime field of $p$ elements for a prime $p>0$, and in this case an entirely different argument seems to be required. ${ }^{2}$

When $\Omega_{0}$ is finite, the group $G_{0}$ is finite, and can be identified at least in certain cases with one of the finite linear groups introduced by Chevalley [1]. The results of this paper exhibit a family of irreducible projective representations of these groups, while in another paper [3], some results on the degrees of these representations are obtained. ${ }^{3}$ The next problem to be investigated in this connection is whether the representations obtained in this paper give all the irreducible projective representations of the groups $G_{0}$.

1. Preliminary results. Familiarity with the paper [2] is assumed.

Received by the editors February 19, 1960.

1 This research was supported in part by the Office of Naval Research.

2 The referee has pointed out that all the irreducible rational projective representations of the group $G$ have been determined by algebraic-geometric methods in Séminaire C. Chevalley, Paris 1956-1958, Exposés 15 and 16. Not all the irreducible representations obtained by Chevalley, however, yield irreducible projective representations upon restriction to the subgroup $G_{0}$. For example, let $G=P S L(2, \Omega)$, where $\Omega$ is an algebraically closed field of characteristic $p>0$. Then $G_{0}=P S L\left(2, \Omega_{0}\right)$. It follows easily from Chevalley's classification of the rational irreducible representations of $S L(2, \Omega)$ (Exposé 20, pp. 20-11 ff.) that $G$ has irreducible rational projective representations of arbitrarily high degree. On the other hand, $G_{0}$, being a finite group, has at most a finite number of inequivalent irreducible projective representations in the field $\Omega$. This result can be proved using the methods of I. Schur, J. Reine Angew. Math. vol. 127 (1904) pp. 20-50, or K. Asano and K. Shoda, Compositio Math. vol. 2 (1935) pp. 230-240, especially $\$ 1$. The full connection between the representations constructed in [2] and those determined in the Séminaire C. Chevalley remains to be determined.

${ }^{3}$ See note added in proof at the end of this paper. 
We begin by recalling, with some minor changes, some of the principal notations in [2].

$\Omega$ algebraically closed field of characteristic $p>7$;

$\Omega_{0}$ prime field in $\Omega$;

$\mathfrak{R}$ Lie algebra of classical type over $\Omega$;

$\mathfrak{S}$ a fixed Cartan subalgebra of $\mathfrak{R}$;

$\alpha, \beta, \cdots$ roots of $\mathfrak{R}$ with respect to $\mathfrak{E}$;

$e(\alpha)$ a fixed basis element for the root space $\mathfrak{R}_{\alpha}, \alpha \neq 0$;

$\Delta=\left\{\alpha_{1}, \cdots, \alpha_{l}\right\}$ a maximal simple system of roots of $\mathfrak{l}$ with respect to $\mathfrak{S}$;

$M$ a fixed irreducible restricted right $\&$-module;

$\lambda$ maximal weight of $M$;

$x_{+}, x_{-}$fixed maximal and minimal vectors, respectively, in $M$.

Our main task is to find a manageable set of generators for $M$. We begin with the remark that for all $v \in M, x, y$ in $\&$, we have

$$
(v x) y-(v y) x=v[x y] \text {, }
$$

because $M$ is a right $\&$-module. From [2] we know that $M$ is spanned over $\Omega$ by $x_{+}$together with vectors

$$
x_{+} e\left(\gamma_{1}\right) \cdots e\left(\gamma_{r}\right), \quad \gamma_{i}<0,
$$

and that for each negative root $\gamma$,

$$
e(\gamma)=\xi\left[\cdots\left[e\left(-\alpha_{i_{1}}\right) e\left(-\alpha_{i_{2}}\right)\right] \cdots e\left(-\alpha_{i_{s}}\right)\right], \quad \alpha_{i_{j}} \in \Delta, \xi \in \Omega .
$$

Combining these facts we conclude that $M$ is spanned over $\Omega$ by $x_{+}$ together with the vector monomials

$$
v=x_{+} e\left(-\alpha_{i_{1}}\right) \cdots e\left(-\alpha_{i_{s}}\right), \quad \alpha_{i_{j}} \in \Delta, s \geqq 0 .
$$

We define the rank of the expression (2) for $v$ to be the ordered $l$ tple of non-negative integers $\left(\rho_{i}\right)=\left(\rho_{1}, \cdots, \rho_{l}\right)$, where $\rho_{i}$ counts the number of indices $j, 1 \leqq j \leqq s$, for which $i_{j}=i$ in (2); in other words $\rho_{i}$ counts the multiplicity of $e\left(-\alpha_{i}\right)$ as a "factor" in (2). We shall call $\rho$ the rank of the vector monomial $v$, and denote it by $\rho(v)$. We shall prove shortly that the rank of $v$ is well defined; until then, when we speak of a vector monomial $v$ of rank $\rho$, we mean that $v$ can be expressed as a vector monomial (2) of rank $\rho$. The maximal vector $x_{+}$is counted as a vector monomial of rank 0 . The utility of the notion of rank comes from the fact that the ranks can be linearly ordered lexicographically. We define $\rho=\left(\rho_{i}\right)<\rho^{\prime}=\left(\rho_{i}^{\prime}\right)$ if $\rho \neq \rho^{\prime}$ and if the first nonvanishing difference $\rho_{i}^{\prime}-\rho_{i}$ is positive. An arbitrary vector $w$ is called a rank vector of rank $\rho$ if $w \neq 0$ and if $w$ is a linear combination of vector monomials of rank $\rho$. Two vectors of the same rank also 
have the same weight in the sense of [2], but the converse is not necessarily true.

We shall denote by $\epsilon_{i}, 1 \leqq i \leqq l$, the $i$ th "unit vector" with a 1 in the $i$ th position and zeros elsewhere. Our first lemma can now be stated as follows.

(1.1) Lemma. Let $v$ be a rank vector of rank $\rho$, and let $\alpha_{i} \in \Delta$. If $v e\left(\alpha_{i}\right) \neq 0$ then $v e\left(\alpha_{i}\right)$ is a rank vector and $\rho\left(v e\left(\alpha_{i}\right)\right)=\rho-\epsilon_{i}$; while if $v e\left(-\alpha_{i}\right) \neq 0, \rho\left(v e\left(-\alpha_{i}\right)\right)=\rho+\epsilon_{i}$.

Proof. It is sufficient to prove the result in case $v$ is a vector monomial (2) of rank $\rho$. We require the fact that because $\Delta$ is a simple system, the difference of two roots $\alpha$ and $\beta$ in $\Delta$ is either zero or is not a root. Therefore

$$
\left[e\left(-\alpha_{k}\right) e\left(\alpha_{i}\right)\right]= \begin{cases}0 & \text { if } k \neq i \\ h_{\alpha_{k}} \in \mathfrak{S} & \text { if } k=i\end{cases}
$$

Now let $v$ be given by (2) and let $v e\left(\alpha_{i}\right) \neq 0$. Then the number of factors $s$ in (2) is not zero, and we have

$$
\begin{aligned}
v e\left(\alpha_{i}\right) & =x_{+} e\left(\alpha_{i}\right) e\left(-\alpha_{i_{1}}\right) \cdots e\left(-\alpha_{i_{s}}\right)+x_{+}\left[e\left(-\alpha_{i_{1}}\right) \cdots e\left(-\alpha_{i_{s}}\right), e\left(\alpha_{i}\right)\right] \\
= & \sum_{k=1}^{s} x_{+} e\left(-\alpha_{i_{1}}\right) \cdots e\left(-\alpha_{i_{k-1}}\right)\left[e\left(-\alpha_{i_{k}}\right) e\left(\alpha_{i}\right)\right] e\left(-\alpha_{i k+1}\right) \cdots e\left(-\alpha_{i_{s}}\right),
\end{aligned}
$$

since $x_{+} e\left(\alpha_{i}\right)=0$. By (3) each term in the last sum is either zero or a multiple of some vector monomial of rank $\rho-\epsilon_{i}$. This proves the first assertion. The second is immediate from the definition of rank.

(1.2) CoRollary. Let $v_{1}$ and $v_{2}$ be rank vectors such that $v_{i} e(\alpha)$ $\neq 0, i=1,2$, for some $\alpha$ such that either $\pm \alpha \in \Delta$. Then $\rho\left(v_{1}\right)<\rho\left(v_{2}\right)$ if and only if $\rho\left(v_{1} e(\alpha)\right)<\rho\left(v_{2} e(\alpha)\right)$.

(1.3) Lemma. Rank vectors of different ranks are linearly independent.

Proof. Suppose there exist rank vectors $w_{1}, \cdots, w_{t}, t>1$, such that $\rho\left(w_{1}\right)>\cdots>\rho\left(w_{t}\right)$ and $w_{1}+\cdots+w_{t}=0$. Because $M$ is irreducible, there exists a sequence of integers $j_{1}, \cdots, j_{n}, 1 \leqq j_{k} \leqq l$, such that

$$
w_{1}^{*}=w_{1} e\left(\alpha_{j_{1}}\right) \cdots e\left(\alpha_{j_{n}}\right)
$$

is a maximal vector, and by the Corollary to Theorem 1 of [2], $w_{1}^{*}$ is a nonzero multiple of $x_{+}$. By Lemma 1.1 it follows that

$$
\rho\left(w_{1}\right)-\epsilon_{j_{1}}-\cdots-\epsilon_{j_{n}}=0 \text {. }
$$


Because $\rho\left(w_{i}\right)<\rho\left(w_{1}\right)$ if $i>1$, we have

$$
w_{i} e\left(\alpha_{j_{1}}\right) \cdots e\left(\alpha_{j_{n}}\right)=0,
$$

and obtain the impossible conclusion

$0=\left(w_{1}+\cdots+w_{t}\right) e\left(\alpha_{j_{1}}\right) \cdots e\left(\alpha_{j_{n}}\right)=w_{1} e\left(\alpha_{j_{1}}\right) \cdots e\left(\alpha_{j_{n}}\right)=w_{1}^{*} \neq 0$.

Therefore our original assumption that a relation $w_{1}+\cdots+w_{t}=0$, $t>1$, could exist was incorrect, and Lemma 1.3 is proved.

(1.4) CoROllary. The rank of a vector monomial is well defined; in other words it is impossible for a vector $v$ to have two expressions (2) of different ranks.

(1.5) Corollary. There is a unique maximal rank $\rho^{*}$. Any vector of rank $\rho^{*}$ is a multiple of the minimal vector $x_{-}$.

Proof. The first statement is immediate by Lemma 1.3 and the fact that $M$ is finite dimensional. For the second, let $v$ be a rank vector which is not a multiple of $x_{-}$. Then by the Corollary to Theorem 1 of [2], this time applied to $\left(0: \mathfrak{u}_{-}\right)$, we conclude that there exist integers $k_{1}, \cdots, k_{8}, 1 \leqq k_{i} \leqq l$, such that

$$
v e\left(-\alpha_{k_{1}}\right) \cdots e\left(-\alpha_{k_{s}}\right)=\xi x_{-}, \quad \xi \neq 0 .
$$

This result combined with Lemma 1.1 implies that $\rho(v) \leqq \rho\left(x_{-}\right)$, and Corollary 1.5 is proved.

The next Lemma is a refinement of Lemma (II. 2.1) of [2]. We recall that the group $G$ is generated by the automorphisms.

$$
\sigma=\sigma(\alpha, \xi)=\exp (a d \xi e(\alpha)), \quad \xi \in \Omega,
$$

where $\alpha$ is a root of $\mathfrak{Q}$ with respect to $\mathfrak{E}$. The projective representation $F$ maps $\sigma$ onto the transformation $F(\sigma)$ given by

$$
x_{+} e\left(-\alpha_{i_{1}}\right) \cdots e\left(-\alpha_{i_{r}}\right) \rightarrow x_{+} e\left(-\alpha_{i_{1}}\right)^{\sigma} \cdots e\left(-\alpha_{i_{r}}\right)^{\sigma}
$$

if $\alpha>0$, and

$$
x_{-} e\left(\alpha_{j_{1}}\right) \cdots e\left(\alpha_{j_{\diamond}}\right) \rightarrow x_{-} e\left(\alpha_{j_{1}}\right)^{\sigma} \cdots e\left(\alpha_{j_{\bullet}}\right)^{\sigma}
$$

if $\alpha<0$.

(1.6) Lemma. Let $v$ be a rank vector in $M$, and let $\sigma=\sigma\left(\alpha_{i}, \xi\right)$, where either $\pm \alpha_{i} \in \Delta$. Then

$$
v F(\sigma)=v+\xi v e\left(\alpha_{i}\right)+v^{*}, \quad \xi \in \Omega,
$$

where $v^{*}$ is a sum of rank vectors all of rank $<\rho(v)-\epsilon_{i}$ if $\alpha_{i} \in \Delta$, and a sum of rank vectors all of rank $>\rho(v)+\epsilon_{i}$ if $-\alpha_{i} \in \Delta$. 
Proof. First suppose that $\alpha_{i} \in \Delta$. We may assume that $v$ is a vector monomial $v=x_{+} e\left(-\alpha_{j_{1}}\right) \cdots e\left(-\alpha_{j_{r}}\right)$. By the argument of Lemma (II.2.1) of [2], we have

$$
v F(\sigma)=v+\xi v e\left(\alpha_{i}\right)+\sum_{k \geq 2} \xi^{k} v_{k},
$$

where $v_{k}, k \geqq 2$, is a linear combination of vectors of the form

$$
x_{+}\left\{e\left(-\alpha_{j_{1}}\right)\left(a d e\left(\alpha_{i}\right)\right) h_{1}\right\} \cdots\left\{e\left(-\alpha_{j_{r}}\right)\left(a d e\left(\alpha_{i}\right)\right)^{h_{r}}\right\},
$$

where $\sum h_{i}=k$. It is immediate by (3) that such a vector is either zero or a rank vector of rank $\rho(v)-k \epsilon_{i}<\rho\left(v e\left(\alpha_{i}\right)\right)$, and upon setting $v^{*}=\sum_{k \geq 2} \xi^{k} v_{k}$, the first assertion of the Lemma is established. The second assertion is proved similarly, starting from a vector $v$ of the form $x_{-} e\left(\alpha_{j_{1}}\right) \cdots e\left(\alpha_{j_{r}}\right), \alpha_{j_{i}} \in \Delta$, and observing that such a vector is a rank vector by Lemma 1.1 and Corollary 1.5. We shall omit the rest of the proof of the second statement.

(1.7) LemMA. Let $w$ be a nonzero vector in $M$ such that $w F(\sigma)=w$ for every $\sigma=\sigma\left(\alpha_{i}, 1\right), \alpha_{i} \in \Delta$. Then $w$ is a maximal vector. Similarly, if $w F(\sigma)=w$ for every $\sigma=\sigma\left(-\alpha_{i}, 1\right), \alpha_{i} \in \Delta$, then $w$ is a minimal vector.

Proof. Again we shall prove only the first assertion. Let $w=w_{1}+\cdots+w_{t}$, where the $w_{i}$ are rank vectors such that $\rho\left(w_{1}\right)>\cdots>\rho\left(w_{t}\right)$ if $t>1$. Let $\sigma=\sigma\left(\alpha_{i}, 1\right)$; then by Lemma 1.6 and the hypothesis of Lemma 1.7 we have

$$
w F(\sigma)=w=w+\sum w_{k} e\left(\alpha_{1}\right)+\sum w_{k}^{*},
$$

where each $w_{k}^{*}$ is a sum of rank vectors of rank less than the rank of $w_{k} e\left(\alpha_{i}\right)$. Then

$$
\sum w_{k} e\left(\alpha_{i}\right)+\sum w_{k}^{*}=0,
$$

and if $w_{1} e\left(\alpha_{i}\right) \neq 0$ then this term is the only rank vector in (5) of rank $\rho\left(w_{1}\right)-\epsilon_{i}$, and we contradict Lemma 1.3. Therefore $w_{1} e\left(\alpha_{i}\right)=0$ for all $\alpha_{i}$ in $\Delta$, and $w_{1}$ is a maximal vector. Because $w_{1}$ has the greatest rank among all the $w_{i}$, we have $w_{2}=\cdots=w_{t}=0$. Hence $w=w_{1}$ is a maximal vector, and Lemma 1.7 is proved.

2. The main theorem. The main theorem of the paper can be stated as follows.

ThEOREM. Let $F$ be the projective representation of $G$ associated with the irreducible restricted $\left\{\right.$-module $M$. Let $G_{0}$ be the subgroup of $G$ generated by the automorphisms $\sigma(\alpha, \xi)$ where $\xi$ is taken from the prime field $\Omega_{0}$ in $\Omega$, and $\alpha$ ranges over the set of roots of $\mathbb{R}$ with respect to a fixed 
Cartan subalgebra $\mathfrak{S}$. Then the restriction $F_{0}$ of $F$ to the subgroup $G_{0}$ is an irreducible projective representation of $G_{0}$. Moreover if $F$ and $F^{\prime}$ are two projective representations of $G$ associated with the irreducible restricted \&-modules $M$ and $M^{\prime}$, and if $S$ is a vector space isomorphism of $M$ onto $M^{\prime}$ such that $S F^{\prime}(\sigma)=F(\sigma) S$ for all generators $\sigma=\sigma(\alpha, \xi)$, $\xi \in \Omega_{0}$, of $G_{0}$, then $M$ and $M^{\prime}$ are $\&$-isomorphic.

Proof. We prove first that $F_{0}$ is irreducible. Let $N \neq 0$ be an $\Omega$-subspace of $M$ which is invariant with respect to all $F(\sigma), \sigma \in G_{0}$. Let $v$ be a nonzero element of $N$, and write $v=\sum_{1}^{t} v_{i}$, where the $v_{i}$ are rank vectors such that $\rho\left(v_{1}\right)>\cdots>\rho\left(v_{t}\right)$. There exist integers $i_{1}, \cdots, i_{s}, 1 \leqq i_{j} \leqq l$, such that $v_{1} e\left(\alpha_{i_{1}}\right) \cdots e\left(\alpha_{i_{s}}\right)$ is a nonzero multiple of $x_{+}$. By successive applications of Lemma 1.6, we obtain

$$
v e\left(\alpha_{i_{1}}\right) \cdots e\left(\alpha_{i_{s}}\right)+v^{*}=\sum_{1}^{t} v_{i} e\left(\alpha_{i_{1}}\right) \cdots e\left(\alpha_{i_{\mathrm{s}}}\right)+v^{*} \in N,
$$

where $v_{1} e\left(\alpha_{i_{1}}\right) \cdots e\left(\alpha_{i_{0}}\right)$ is the unique term of highest rank in the expression. It follows that

$$
\sum_{2}^{t} v_{i} e\left(\alpha_{i_{1}}\right) \cdots e\left(\alpha_{i_{s}}\right)+v^{*}=0
$$

and that $x_{+}=\xi v_{1} e\left(\alpha_{i_{1}}\right) \cdots e\left(\alpha_{i_{s}}\right) \in N$. Similarly $x_{-} \in N$.

By the same reasoning we see that for every rank vector in $M$ of the form

$$
v=x_{-} e\left(\alpha_{i_{1}}\right) \cdots e\left(\alpha_{i_{s}}\right),
$$

$N$ contains a vector $v+v^{*}$ where $v^{*}$ is a sum of rank vectors all of rank less than $\rho(v)$. The space $M$ has a basis $\left\{v_{i}\right\}$ consisting of vectors of the form (6), and corresponding to this basis we have a set of vectors $\left\{w_{i}=v_{i}+v_{i}^{*}\right\}$ in $N$. We prove that the vectors $w_{i}$ are linearly independent. If we have a relation of linear dependence

$$
\sum \xi_{i} w_{i}=0,
$$

with some $\xi_{i} \neq 0$, then there will exist vectors $w_{i_{j}}=v_{i_{j}}+v_{i_{j}}^{*}, 1 \leqq j \leqq r$, with nonzero coefficients $\xi_{i_{j}}$, and with the rank of the $v_{i_{j}}$ as large as possible. Applying Lemma 1.3, we obtain $\sum_{1}^{r} \xi_{i_{j}} v_{i_{j}}=0$, contrary to the assumption that the $\left\{v_{i}\right\}$ are linearly independent. Therefore $N$ contains a basis of $M$, and we have proved that $M$ is irreducible relative to $G_{0}$.

In order to prove the second assertion, it is sufficient by Theorem 1 of [2], to prove that $M$ and $M^{\prime}$ have the same maximal weight. From the hypothesis that 


$$
S F^{\prime}(\sigma)=F(\sigma) S, \quad \sigma \in G_{0},
$$

we obtain

$$
x_{+} S F^{\prime}(\sigma)=x_{+} F(\sigma) S=x_{+} S, \quad \sigma=\sigma\left(\alpha_{i}, 1\right), \quad \alpha_{i} \in \Delta .
$$

By Lemma 1.7 applied to $M^{\prime}$, we see that $x_{+} S$ is a maximal vector in $M^{\prime}$, and by a similar argument, $x_{-} S$ is a minimal vector in $M^{\prime}$. The concept of rank is meaningful in both $M$ and $M^{\prime}$, and we shall prove, after some preliminary steps, that $S$ preserves rank.

(2.1) Lemma. $\rho\left(x_{-} S\right)=\rho\left(x_{-}\right)$.

Proof. Suppose first that $\rho\left(x_{-}\right)<\rho\left(x_{-} S\right)$. We shall then prove that if $v$ is an arbitrary rank vector in $M$, and if $v S=\sum_{j \geq 0} w_{j}^{\prime}$, where the $w_{j}^{\prime}$ are rank vectors in $M^{\prime}$ such that $\rho\left(w_{0}^{\prime}\right)<\rho\left(w_{1}^{\prime}\right)<\cdots$, then $\rho(v)<\rho\left(w_{0}^{\prime}\right)$. We have the result for the vector $x_{-}$of maximal rank, and we may assume as an induction hypothesis that the result is true for all $v_{1}$ such that $\rho\left(v_{1}\right)>\rho(v)$. We may also assume that $w_{0}^{\prime}$ is not a minimal vector in $M^{\prime}$, otherwise $v$ is a minimal vector in $M$, and the result is known. There exists a root $\alpha_{i} \in \Delta$ such that $w_{0}^{\prime} e\left(-\alpha_{i}\right) \neq 0$. Applying (7) and Lemma 1.6 to $v$ and $\sigma=\sigma\left(-\alpha_{i}, 1\right)$, we obtain

$$
\left(v+v e\left(-\alpha_{i}\right)+v^{*}\right) S=v S+\sum w_{j}^{\prime} e\left(-\alpha_{i}\right)+\sum\left(w_{j}^{\prime}\right)^{*},
$$

where $v^{*}$ is a sum of rank vectors all of rank $>\rho(v)+\epsilon_{i}$, while $w_{0}^{\prime} e\left(-\alpha_{i}\right)$ is the unique term of minimal rank on the right-hand side after $v S$ has been cancelled. Applying the induction hypothesis to $v e\left(-\alpha_{i}\right)$ and $v^{*}$, we conclude that $\rho(v)+\epsilon_{i}<\rho\left(w_{0}^{\prime} e\left(-\alpha_{i}\right)\right)$, and it follows that $\rho(v)<\rho\left(w_{0}^{\prime}\right)$ as required. The result just established, however, implies that $\rho\left(x_{+}\right)<\rho\left(x_{+} S\right)$ which is impossible. Therefore the hypothesis that $\rho\left(x_{-}\right)<\rho\left(x_{-} S\right)$ is untenable, and $\rho\left(x_{-}\right) \geqq \rho\left(x_{-} S\right)$. Similarly $\rho\left(x_{-}\right) \leqq \rho\left(x_{-} S\right)$, and Lemma 2.1 is proved.

(2.2) Lemma. For any rank vector $v$ in $M, v S=\sum_{j \geq 0} w_{j}^{\prime}$, where the $w_{j}^{\prime}$ are rank vectors in $M^{\prime}$ such that $\rho(v) \leqq \rho\left(w_{0}^{\prime}\right)<\rho\left(w_{1}^{\prime}\right)<\cdots$.

Proof. By Lemma 2.1, the result is true for $v$ of maximal rank, and we may assume it for all $v_{1}$ such that $\rho\left(v_{1}\right)>\rho(v)$. As in the proof of Lemma 2.1, find $\alpha_{i} \in \Delta$ such that $w_{0}^{\prime} e\left(-\alpha_{i}\right) \neq 0$, and write down the equation (8). Again we may apply the induction hypothesis to the left side, to conclude that $\rho(v)+\epsilon_{i} \leqq \rho\left(w_{0}^{\prime} e\left(-\alpha_{i}\right)\right)$ and hence $\rho(v) \leqq \rho\left(w_{0}^{\prime}\right)$.

Similarly we can prove that $v S=\sum_{j \geq 0} w_{j}^{\prime \prime}$, where the $w_{j}^{\prime \prime}$ are rank vectors in $M^{\prime}$ such that $\rho(v) \geqq \rho\left(w_{0}^{\prime \prime}\right)>\cdots$. Combining our results, and applying Lemma 1.3, we deduce that for all rank vectors $v$ in $M, v S$ is a rank vector in $M^{\prime}$ and $\rho(v S)=\rho(v)$.

Now we can prove that $M$ and $M^{\prime}$ have the same maximal weight. 
First suppose $x_{+} e\left(-\alpha_{i}\right) \neq 0$. Then from $x_{+} F\left(\sigma\left(-\alpha_{i}, 1\right)\right) S$ $=x_{+} S F^{\prime}\left(\sigma\left(-\alpha_{i}, 1\right)\right)$ and Lemma 1.6 we obtain

$$
\left(x_{+}+x_{+} e\left(-\alpha_{i}\right)+x_{+}^{*}\right) S=x_{+} S+x_{+} S e\left(-\alpha_{i}\right)+\left(x_{+} S\right)^{*} .
$$

Because $S$ preserves rank we can apply Lemma 1.3 to get

$$
x_{+} e\left(-\alpha_{i}\right) S=\left(x_{+} S\right) e\left(-\alpha_{i}\right) .
$$

Now apply $F^{\prime}\left(\sigma\left(\alpha_{i}, 1\right)\right)$ to both sides of (9). This yields

$$
x_{+} e\left(-\alpha_{i}\right) F\left(\sigma\left(\alpha_{i}, 1\right)\right) S=\left(x_{+} S\right) e\left(-\alpha_{i}\right) F^{\prime}\left(\sigma\left(\alpha_{i}, 1\right)\right),
$$

and we obtain

$$
\begin{aligned}
& {\left[x_{+} e\left(-\alpha_{i}\right)+x_{+} e\left(-\alpha_{i}\right) e\left(\alpha_{i}\right)+\left(x_{+} e\left(-\alpha_{i}\right)\right)^{*}\right] S} \\
& \quad=x_{+} S e\left(-\alpha_{i}\right)+x_{+} \operatorname{Se}\left(-\alpha_{i}\right) e\left(\alpha_{i}\right)+\left(x_{+} S e\left(-\alpha_{i}\right)\right)^{*} .
\end{aligned}
$$

Equating terms of equal rank we have

$$
\lambda\left(h_{\alpha_{i}}\right) x_{+} S=\lambda^{\prime}\left(h_{\alpha_{i}}\right) x_{+} S,
$$

where $\lambda$ and $\lambda^{\prime}$ are the maximal weights of $M$ and $M^{\prime}$ respectively. Finally suppose $x_{+} e\left(-\alpha_{i}\right)=0$; then $\lambda\left(h_{\alpha_{i}}\right)=0$, and we obtain $x_{+} \operatorname{Se}\left(-\alpha_{i}\right)=0$, so that $\lambda^{\prime}\left(h_{\alpha_{i}}\right)=0$. We have proved that $\lambda\left(h_{\alpha_{i}}\right)=\lambda^{\prime}\left(h_{\alpha_{i}}\right)$ for all $\alpha_{i} \in \Delta$. Because the $h_{\alpha_{i}}$ span $\mathfrak{S}$, we conclude that $\lambda=\lambda^{\prime}$, and the theorem is proved.

Added in proof. We take this opportunity to correct an error in [3]. In that paper the assertion on p. 141 " $m_{\alpha}=m_{\alpha}^{\prime}$ " following the proof of Theorem 2 is false, and invalidates the subsequent construction of the example, although formula (9) is correct as it stands. An example to show that Weyl's formula does not always hold at characteristic $p$ can be constructed as follows. Let $\mathfrak{R}$ be the simple Lie algebra of type $A_{2}$ over $\Omega$ of characteristic $p \geqq 5$, viewed as the algebra of linear transformations of trace zero on a 3 -dimensional vector space $M_{0}$. Let $\alpha_{1}$ and $\alpha_{2}$ be a maximal simple system of roots with respect to a Cartan subalgebra, and let $v_{0}$ be a maximal vector in $M_{0}$ such that $v_{0} e_{\alpha_{2}} \neq 0$. Let $v_{0}^{p}=v_{0} \otimes \cdots \otimes v_{0}$ ( $p$ times) in the tensor algebra $T\left(M_{0}\right)$ on $M_{0}$. Then $v=v_{0}^{p} e_{-\alpha_{2}}$ is a maximal vector in $T\left(M_{0}\right)$ of weight $\lambda$ such that $\lambda\left(h_{\alpha_{1}}\right)=1, \lambda\left(h_{\alpha_{2}}\right)=p-2$. The irreducible restricted \&module $M$ whose maximal weight is $\lambda$ is a composition factor of $v \mathfrak{u}$, and since $v \mathfrak{U}$ is contained in the space of symmetric $p$-tensors, we have $\operatorname{dim} M \leqq(p+1)(p+2) / 2$. On the other hand, formula (9) of [3] asserts that for the associated module $V$ of $M$ we have

$$
\operatorname{dim} V=p^{2}-1>\operatorname{dim} M
$$

if $p \geqq 5$. 


\section{REFERENCES}

1. C. Chevalley, Sur certains groupes simples, Tôhoku Math. J. vol. 7 (1955) pp. 14-66.

2. C. W. Curtis, Representations of Lie algebras of classical type with applications to linear groups, J. Math. Mech. vol. 9 (1960) pp. 307-326.

3. - On the dimensions of the irreducible modules of Lie algebras of classical type, Trans. Amer. Math. Soc. vol. 96 (1960) pp. 135-142.

UNIVERSITY OF WISCONSIN 